\title{
Phytochemicals and biological studies of plants from the genus Balanophora
}

\author{
Xiaohong Wang, Zizhen Liu, Wenlin Qiao, Ruiyang Cheng, Bin Liu and Gaimei She*
}

\begin{abstract}
This review focus on the phytochemical progress and biological studies of plants from the genus Balanophora (Balanophoraceae) over the past few decades, in which most plants growth in tropical and subtropical regions of Asia and Oceania, and nearly 20 species ranged in southwest China. These dioeciously parasitic plants are normally growing on the roots of the evergreen broadleaf trees, especially in the family of Leguminosae, Ericaceae, Urticaceae, and Fagaceae. The plants are mainly used for clearing away heat and toxic, neutralizing the effect of alcoholic drinks, and as a tonic for the treatment of hemorrhoids, stomachache and hemoptysis. And it has been used widely throughtout local area by Chinese people.

Cinnamic acid derivative tannins, possessing a phenylacrylic acid derivative (e. g. caffeoyl, coumaroyl, feruloyl or cinnamoyl), which connected to the $C(1)$ position of a glucosyl unit by O-glycosidic bond, are the characteristic components in genus Balanophora. In addition, several galloyl, caffeoyl and hexahydroxydiphenoyl esters of dihydrochalcone glucosides are found in B. tobiracola, B. harlandii, and B. papuana. Other compounds like phenylpropanoids, flavonoids, terpenoids and sterols are also existed. And their biological activities, such as radical scavenging activities, HIV inhibiting effects, and hypoglycemic effects are highlighted in the review.
\end{abstract}

\section{Review}

\section{Introduction}

Balanophora is a genus belonging to the family Balanophoraceae which possesses about 120 species all over the world. Many of them distribute in tropical and subtropical regions of Asia and Oceania, and nearly 20 species are widely ranged in southwest of China [1,2]. These types of dioeciously parasitic plants are normally growing on the roots of the evergreen broadleaf trees, especially in the family of Leguminosae, Ericaceae, Urticaceae, and Fagaceae [1,3-5]. Species in this genus have miscellaneous biological properties such as clearing away heat and toxic, neutralizing the effect of alcoholic drinks, and used as a tonic for the treatment of hemorrhoids, stomachache and hemoptysis by local people in China. The plants of genus Balanophora have been recorded in the Compendium of Materia Medica and were called 'Gecaihua' or 'Geru' [3,5,6].

\footnotetext{
*Correspondence: shegaimei@126.com

Department of Traditional Chinese Medicine, School of Chinese Pharmacy, Beijing University of Chinese Medicine, Beijing 100102, China
}

Phytochemical investigation of genus Balanophora showed that the hydrolysable tannins are rich in species, particularly the ellagitannins. Structurally, cinnamic acid derivative tannins are the chemically-characterised constituents. It possesses a phenylacrylic acid derivative (e. g. caffeoyl, coumaroyl, feruloyl or cinnamoyl), which connected to the $C(1)$ position of a glucosyl unit by $O$-glycosidic bond, and part of galloyl, caffeoyl and hexahydroxydiphenoyl (HHDP) are linked with the $\mathrm{C}(1)$, $C(3), C(4)$ and / or $C(6)$ positions through aromatic ester bonds. 1-O-caffeoyl-(4-O-galloyl)- $\beta$-D-glucopyranose (2) and 1-O-caffeoyl-(6-O-galloyl)- $\beta$-D-glucopyranose (3), which were first reported from $B$. harlandii in 2000 by Teng et al., have the analogous feature [7]. In addition, several galloyl, caffeoyl and hexahydroxydiphenoyl esters of dihydrochalcone glucosides (43-51) found in B. tobiracola, $B$. harlandii, and $B$. papuana, displayed obviously radical-scavenging activities, inhibiting HIV, as well as hypoglycemic effects, etc. [1,3,6,8-10]. Aside from those, the galloyl, HHDP and their derivatives linking the glucosyl unit at $C(1), C(3), C(4)$ and / or $C(6)$ positions through ester bonds are major constituents in the genus Balanophora [1,2,11-18]. 
In this review, we mainly summarize the study progress of phytochemical over the past decades and list the entire chemical compounds isolated from the genus Balanophora. Meanwhile, the biological activities of the plant extracts and chemical constituents are highlighted as well.

\section{The phytochemical studies of genus Balanophora}

Phytochemical Constituents. - There are several sorts of chemical constituents existing in genus Balanophora. The reported diverse chemical structures of tannins substances owe to their functional groups and the attended modes as well as linked locations of its substituting groups. Extensive studies have led to the identification of many other compounds, such as $\mathrm{C}_{6}-\mathrm{C}_{3}$ and $\mathrm{C}_{6}-\mathrm{C}_{3}-\mathrm{C}_{6}$ constituents, terpenoids, sterols and so forth. The structures of compounds in the Figure [see Additional file 1] and their names are numbered 1-149, the corresponding plant sources and references are listed in the Table 1.

2.1 Tannins. There are abundant and varied tannins (1-61) in the genus Balanophora. The hydrolyzable tannins - one and / or several galloyls, HHDP, caffeoyl and coumaroyl groups attached to one glucosyl unit by ester linkage - were reported as predominant components from this genus.

Cinnamic acid derivative tannins were found as characteristic components in this genus. They have a caffeoyl, feruloyl, coumaroyl or cinnamoyl group connected at $C(1)$ position in a glucosyl moiety by acyl $O$-glycosidic linkage. Positions $C(3)$ and $C(4)$ in glucosyl moiety are usually attached to a galloyl, together with a HHDP group often linked to the $C(4)$ and $C(6)$ positions. The $C(2)$ position often has a unsubstituted hydroxyl group. In addition to the aforementioned connection types, the 1,2-di-, 1, 3-di- and 1, 2, 6-tri-caffeoyl compounds (e.g. compounds 5, 6 and 13), which were also from the genus Balanophora, were regarded as cinnamic acid derivative tannins $[14,17]$. Up to now, compounds 1-13, 28, 30-31, 33 - 41 from B. spicata, B. fungosa, B. polyandra, B. laxiflora, B. harlandii and B. japonica are fall into this category $[1,5,7,8,14-21]$. To the best of our knowledge, this kind of compounds did not discovered in other families.

The caffeoyl group is connected to the $C(1)$ position in compounds $1-4,7-12$, and the galloyl is linked to the $C(3), C(4)$ and $C(6)$ of the glucosyl moiety. As for the HHDP groups, it was mostly joint to $C(4)$ and $C(6)$ $[1,5,7,14,16-18]$. Compounds $28-30$, isolated from the plants B. polyandra and B. japonica, have a caffeoyl or galloyl group at $C(1)$ position and a 1, 1'- (3, 3', 4, 4'-tetrahydroxy) dibenzofurandicarboxyl group attached to the $C(4)$ and $C(6)$ positions of the glucosyl unit [21]. The compounds 31-34 isolated from B. japonica contain an oxidized-HHDP group named brevifolincarboxylate
[22]. For 1-O- $(E)$-caffeoyl-6-O- $(S)$-brevifolincarboxyl$\beta$-D-glucopyranose (31) and 1, 3-di-O-galloyl-6-O- $(S)$ brevifolincarboxyl- $\beta$-D-glucopyranose (32), their $C(6)$ of glucosyl moiety connect to a brevifolincarboxylate group. The HMBC spectrum and phenylglycine methyl ester (PGME) methods played important roles in the elucidatation of the chemical structures of 31 and 32 [22]. Balanophotannin E (32) exhibited strong cytotoxicity to HepG2 cells, which is equivalent to that of cisplatin. Methylated balanophotannins F (33) by alkaline hydrolysis obtained the heptamethyl derivative. Balanophotannins G (34) has a caffeoyl, 1, 6-diacylated glucopyranosyl and an aromatic lactones structural unit. In particular, the $C(2)$ and $C(5)$ positions in compound 34 present a $-\mathrm{COO}^{-} \mathrm{K}^{+}$group correspondingly. And the inorganic element $\mathrm{K}$ was determined by negative FAB-MS and HR-ESI-MS [22]. Balapolyphorins A (35) was also cinnamic acid derivative tannin with a balapolyphoroyl group at $C(6)$ position in glucosyl unit to form aryl ester and a caffeoyl group to the $\mathrm{C}(1)$ by acyl $O$-glycosidic linkage [15]. Compounds 36-41 take the characteristics that a coumaroyl or cinnamoyl is connected to anomeric carbon by $\mathrm{O}$-glycosidic bond, and several galloyl and HHDP groups are linked with the $C(3), C(4)$ and / or $C(6)$ positions in glucosyl moiety to form sugar aryl ester linkages $[1,5,14,16-18]$.

Another attended mode was that the $\mathrm{OH}$ group at $\mathrm{C}(1)$ position in glucosyl unit connected with a galloyl by one galloyl ester glycoside in compounds 14-27, 29 and 42 $[1,5,12-15,20,21]$. And other positions' $\mathrm{OH}$ groups are substituted by galloyl, HHDP and caffeoyl group. Among them, the $\mathrm{C}-\mathrm{O}(\mathrm{C}-3)$ in 18 and $\mathrm{C}-6$ in 27 are attached to a caffeoyl group. Additional substituent modes, such as 1 , 3-di-, 1, 3, 4-tri-, 1, 3, 4, 6-tetra-galloyl groups and 4, 6-HHDP moieties are in existence as well. The balapolyphorins B (42) emerges two galloyl groups in C(1), $\mathrm{C}(6)$ and linked a caffeoyl in C(4) $[1,5,14,17]$.

Galloyl, caffeoyl and hexahydroxydiphenoyl esters of dihydrochalcone glucosides (43-51) were found in the plants of B. tobiracola, B. harlandii and B. papuana $[1,8,9]$. Compounds $43-48$ are structurally characterized by one or ones of caffeoyl, galloyl or HHDP group ester hold to the $C\left(2^{\prime \prime}\right), C\left(3^{\prime \prime}\right), C\left(4^{\prime \prime}\right)$ and $C\left(6^{\prime \prime}\right)$ positions in glucosyl unit of 3-hydroxyphloretin-4'-O- $\beta$-glucoside $[1,8]$. Papuabalanols A (50) and papuabalanols B (51) were two new dehydrohexahydroxydiphenoyl (DHHDP) esters of dihydrochalcone glycosides isolated from the ethyl acetate extract of $B$. papuana, with the DHHDP skeletons located in $C(4 ")$ and $C\left(6^{\prime \prime}\right)$ positions of the glucosyl units [9].

According to the ${ }^{1} \mathrm{H}$ - and ${ }^{13} \mathrm{C}$-NMR spectrum of compounds $52-58$, it showed the presence of $1 / 2 \alpha-$ and $1 / 2 \beta$-D-glucopyranosyl units. The duplication $(\alpha / \beta$ forms) of the signals revealed by glucosyl unit suggested 


\section{Table 1 Chemical constituents from genus balanophora}

\begin{tabular}{|c|c|c|c|}
\hline No. & Name & Source & Ref. \\
\hline 1 & 1-O-(E)-caffeoyl-3-O-galloyl-B-D-glucopyranose & $\begin{array}{l}\text { B. harlandii, B. spicata, B. japonica, B. fungosa, } \\
\text { B. laxiflora }\end{array}$ & {$[1,8,14,16,19,20$} \\
\hline 2 & 1-O-caffeoyl-(4-O-galloyl)- $\beta$-D-glucopyranose & B. harlandii, B. japonica & {$[7,14]$} \\
\hline 3 & 1-O-caffeoyl-(6-O-galloyl)- $\beta$-D-glucopyranose & B. harlandii, B. japonica & {$[7,14]$} \\
\hline 4 & 1-O-(E)-caffeoyl-4,6-(S)-HHDP-ß-D-glucopyranose & B. japonica, B. fungosa, B. laxiflora & {$[14,16,20]$} \\
\hline 5 & 1,2-di-O-(E)-caffeoyl- $\beta$-D-glucopyranose & B. japonica & [14] \\
\hline 6 & 1,3-di-O-(E)-caffeoyl- $\beta$-D-glucopyranose & B. japonica & [14] \\
\hline 7 & 1-O-(E)-caffeoyl-3,4-di-O-galloyl- $\beta$-D-glucopyranose & B. japonica & [14] \\
\hline 8 & 1-O-(E)-caffeoyl-4,6-di-O-galloyl- $\beta$-D-glucopyranose & B. laxiflora, B. japonica, B. polyandra & {$[5,14,15]$} \\
\hline 9 & 1-O-(E)-caffeoyl-3-O-galloyl-4,6-(S)-HHDP- $\beta$-D-glucopyranose & B. laxiflora, B. japonica, B. fungosa & {$[5,14,16,19]$} \\
\hline 10 & 1,3-di-O-(E)-caffeoyl-4,6-(S)-HHDP- $\beta$-D-glucopyranose & B. laxiflora, B. japonica & {$[5,14]$} \\
\hline 11 & 1,3-di-O-caffeoyl-4-O-galloyl- $\beta$-D-glucopyranose & B. japonica & {$[14,17]$} \\
\hline 12 & 1-O-(E)-caffeoyl-3,4,6-tri-galloyl- $\beta$-D-glucopyranose & B. fungosa & {$[16,19]$} \\
\hline 13 & 1,2,6-tri-O-caffeoyl- $\beta$-D-glucopyranose & B. japonicaB. laxiflora & {$[14,17]$} \\
\hline 14 & 1,3-di-O-galloyl-4,6-(S)-HHDP- $\beta$-D-glucopyranose & B. laxiflora, B. polyandra, B. japonica & {$[5,13-15,20]$} \\
\hline 15 & 1,3-di-O-galloyl- $\beta$-D-glucopyranose & B. harlandii, B. laxiflora, B. japonica, B. polyandra & {$[1,5,14,15]$} \\
\hline 16 & 1-O-galloyl- $\beta$-D-glucopyranose & B. polyandra & {$[15]$} \\
\hline 17 & 1,3,4-tri-O-galloyl- $\beta$-D-glucopyranose & B. harlandii, B. japonica & {$[1,14]$} \\
\hline 18 & 3-O-(E)-caffeoyl-1-O-galloyl-B-D-glucopyranose & B. polyandra & [15] \\
\hline 19 & 1,2,6-tri-O-galloyl- $\beta$-D-glucopyranose & B. polyandra & {$[15]$} \\
\hline 20 & 1,4-di- O-galloyl- $\beta$-D-glucopyranose & B. japonica & [14] \\
\hline 21 & 1,2,4-tri- O-galloyl- $\beta$-D-glucopyranose & B. japonica & [14] \\
\hline 22 & 1,2,6-tri-O-galloyl- $\beta$-D-glucopyranose & B. laxiflora, B. japonica & {$[5,14]$} \\
\hline 23 & 1,4,6-tri-O-galloyl- $\beta$-D-glucopyranose & B. japonica & [14] \\
\hline 24 & 1,3,4,6-tetra-O-galloyl- $\beta$-D-glucopyranose & B. japonica & [14] \\
\hline 25 & 1-O-galloyl-4,6-[(S)-HHDP]- $\beta$-D-glucopyranose & B. japonica, B. polyandra & {$[14,15]$} \\
\hline 26 & 3-O-(E)-caffeoyl-1,4-di-O-galloyl- $\beta$-D-glucopyranose & B. japonica & [14] \\
\hline 27 & 6-O-(E)-caffeoyl-1,3,4-tri-O-galloyl- $\beta$-D-glucopyranose & B. japonica & [14] \\
\hline 28 & $\begin{array}{l}\text { 1-O-[(E)-caffeoyl]-4,6-[1,1'-(3,3',4,4'- } \\
\text { tetrahydroxydibenzofurandicarboxyl)]-B-D-glucopyranose }\end{array}$ & B. polyandra, B. japonica & {$[15,21]$} \\
\hline 29 & balanophotannins A & B. japonica & {$[21]$} \\
\hline 30 & balanophotannins $C$ & B. japonica & {$[21]$} \\
\hline 31 & 1-O-(E)-caffeoyl-6-O-(S)-brevifolincarboxyl- $\beta$-D-glucopyranose & B. japonica & [22] \\
\hline 32 & 1,3-di-O-galloyl-6-O-(S)-brevifolincarboxyl- $\beta$-D-glucopyranose & B. japonica & [22] \\
\hline 33 & balanophotannins F & B. japonica & [22] \\
\hline 34 & balanophotannins $\mathrm{G}$ & B. japonica & [22] \\
\hline 35 & balapolyphorin A & B. polyandra & [15] \\
\hline 36 & 1-O-(E)-cinnamoyl-3-galloyl-4,6-(S)-HHDP- $\beta$-D-glucopyranose & B. fungosa & [16] \\
\hline 37 & 1-O-(E)-cinnamoyl-4-galloyl- $\beta$-D-glucopyranose & B. fungosa & [16] \\
\hline 38 & 1-O-(E)-coumaroyl-4,6-(S)-HHDP-ß-D-glucopyranose & B. japonica, B. fungosa & {$[14,16]$} \\
\hline 39 & 1-O-(E)-coumaroyl-3-galloyl-4,6-(S)-HHDP- $\beta$-D-glucopyranose & B. fungosa & [16] \\
\hline 40 & 1-O-(E)-coumaroyl-3,4,6-tri-galloyl- $\beta$-D-glucopyranose & B. fungosa & [16] \\
\hline 41 & 1-O-(E)-p-coumaroyl-3-O-galloyl- $\beta$-D-glucopyranose & B. harlandii & {$[1]$} \\
\hline 42 & balapolyphorin B & B. polyandra & [15] \\
\hline 43 & 3-hydroxyphloretin 4'-O-(6"-O-galloyl)- $\beta$-D-glucoside & B. harlandii, B. tobiracola & {$[1,8]$} \\
\hline
\end{tabular}




\section{Table 1 Chemical constituents from genus balanophora (Continued)}

\begin{tabular}{|c|c|c|c|}
\hline 44 & 3-hydroxyphloretin 4'-O-(3",4"-di-O-galloyl)-ß-D-glucoside & B. tobiracola & [8] \\
\hline 45 & 3-hydroxyphloretin 4'-O-(4",6"-di-O-galloyl)-ß-D-glucoside & B. tobiracola & [8] \\
\hline 46 & 3-hydroxyphloretin 4'-[4",6"-di-O-(S)-HHDP- $\beta$-D-glucoside] & B. harlandii, B. tobiracola & {$[1,8]$} \\
\hline 47 & 3-hydroxyphloretin 4'-O-[3"-O-galloyl-4", 6"-O-(S)-HHDP]-ß-D-glucoside & B. tobiracolaB. harlandii & [1] \\
\hline 48 & 3-hydroxyphloretin 4'-O-[3"-O-caffeoyl-4", 6"-O-(S)-HHDP]- $\beta$-D-glucoside & B. tobiracola & [8] \\
\hline 49 & phloretin 4'-O-[3'-O-galloyl-4',6'-O-(S)-HHDP]- $\beta$-D-glucoside & B. tobiracola & [8] \\
\hline 50 & papuabalanols A & B. papuana & [9] \\
\hline 51 & papuabalanols B & B. papuana & [9] \\
\hline 52 & 3-O-galloyl-4,6-[(S)-HHDP]- $\beta$-D-glucopyranose & B. japonica, B. polyandra & {$[14,15]$} \\
\hline 53 & 3-O-galloyl- $\beta$-D-glucopyranose & B. harlandii, B. laxiflora, B. japonica, B. polyandra & {$[1,5,14,15]$} \\
\hline 54 & 6-O-galloyl- $\beta$-D-glucopyranose & B. laxiflora & [5] \\
\hline 55 & 3-O-[(E)-caffeoyl]-4-O-galloyl- $\beta$-D-glucopyranose & B. japonica & [14] \\
\hline 56 & 6-O-(E)-caffeoyl- $\beta$-D-glucopyranose & B. japonica & [14] \\
\hline 57 & 2,6-di-O-galloyl-D-glucopyranose & B. japonica & [14] \\
\hline 58 & 3,4,6-tri-O-galloyl-D-glucopyranose & B. japonica & [14] \\
\hline 59 & gallic acid & $\begin{array}{l}\text { B. laxiflora, B. harlandii, B. polyandra, } \\
\text { B. simaoensis, B. japonica B. fungosa }\end{array}$ & {$[5,7,15,23,24]$} \\
\hline 60 & methyl gallate & B. polyandra & [15] \\
\hline 61 & ellagic acid & B. simaoensis & [23] \\
\hline 62 & methyl $p$-cumarate & B. japonica & [25] \\
\hline 63 & coumaric acid & B. laxiflora, B. fungosa, B. abbreviata & {$[5,16,26]$} \\
\hline 64 & p-hydroxycinnamic- $\beta$-D-glucopyranose & B. fungosa & [16] \\
\hline 65 & caffeic acid & B. laxiflora, B. simaoensis, B. japonica & {$[5,23-25]$} \\
\hline 66 & caffeic acid methyl ester & B. japonica & {$[25]$} \\
\hline 67 & caffeic acid ethyl ester & B. spicata, B. Japonica & {$[19,25]$} \\
\hline 68 & methylconiferin & B. polyandra, B. simaoensis, B. involucrata & {$[15,27-29]$} \\
\hline 69 & butylconiferin & B. simaoensis & [28] \\
\hline 70 & $\begin{array}{l}\text { coniferin (= 4-(3-hydroxyprop-1-en-1-yl)-2- } \\
\text { methoxyphenyl- } \beta \text {-D-glucopyranoside) }\end{array}$ & $\begin{array}{l}\text { B. harlandii, B. laxiflora, B. polyandra, B. polyandra, } \\
\text { B. fungosa, B. involucrata, B. japonica, B. abbreviata, } \\
\text { B. involucrata }\end{array}$ & $\begin{array}{l}{[1,5,15,16,18} \\
25-27,29]\end{array}$ \\
\hline 71 & coniferin aldehyde- $\beta$-D-glucoside & B. latisepala & {$[30]$} \\
\hline 72 & 4-O-(6'-O-p-counmaroyl- $\beta$-D-glucopyranosyl)-coniferyl aldehyde & B. latisepala & [30] \\
\hline 73 & ferulyl aldehyde & B. papuana, B. japonica & {$[9,25]$} \\
\hline 74 & ferulyl aldehyde- $\beta$-D-glucoside & B. japonica & [25] \\
\hline 75 & methyl cinnamate & B. polyandra, B. fungosa & {$[16,31]$} \\
\hline 76 & cinnamic acid & B. polyandra, B. abbreviata & {$[16,26]$} \\
\hline 77 & coniferyl aldehyde & B. abbreviata & [26] \\
\hline 78 & methyl caffeate & B. harlandii & {$[1]$} \\
\hline 79 & cinnamoyl- $\beta$-D-glucopyranose & B. polyandra & {$[16,31]$} \\
\hline 80 & p-hydroxycinnamoyl- $\beta$-D-glucopyranose & B. polyandra, B. laxiflora & [16] \\
\hline 81 & 4'-hydroxy-3'-methoxycinnamoyl- $\beta$-D-glucopyranose & B. polyandra & [16] \\
\hline 82 & balaxiflorin B = (6'-O-(E)-caffeoyl coniferin) & B. laxiflora & [5] \\
\hline 83 & 2-O-(E)-caffeoyl-1-O-p-(E)-coumaroyl- $\beta$-D-glucopyranose & B. japonica & [14] \\
\hline 84 & 1-O-(E)-caffeoyl- $\beta$-D-glucopyranose & B. laxiflora, B. harlandii, B. polyandra, B. laxiflora & {$[1,5,7,14-16,20,28]$} \\
\hline 85 & 1-O-(E)-caffeoyl- $\beta$-gentiobiose & B. japonica, B. polyandra & {$[14,15]$} \\
\hline 86 & 1-O-(3'-O- $\beta$-D-glucopyranosyl)-(E)-caffeoyl- $\beta$-D-glucopyranose & B. japonica & [14] \\
\hline
\end{tabular}


Table 1 Chemical constituents from genus balanophora (Continued)

\begin{tabular}{|c|c|c|c|}
\hline 87 & balajaponin B & B. japonica & {$[32]$} \\
\hline 88 & balajaponin C & B. japonica & {$[32]$} \\
\hline 89 & balajaponin E & B. japonica & {$[32]$} \\
\hline 90 & balajaponin D & B. japonica & {$[32]$} \\
\hline 91 & isolariciresinol & B. laxiflora, B. abbreviata & {$[5,26]$} \\
\hline 92 & isolariciresinol-4-O- $\beta$-D-glucoside & B. laxiflora, B. polyandra, B. abbreviata & {$[5,15,26]$} \\
\hline 93 & (+)-pinoresinol-di-O- $\beta$-D-glucopyranoside & B. spicata, B. japonica & {$[12,21]$} \\
\hline 94 & (+)-pinoresinol-O- $\beta$-D-glucopyranoside & B. laxiflora, B. japonica & {$[5,21]$} \\
\hline 95 & $(+)$-pinoresinol & B. polyandra, B. abbreviata & {$[16,26,33]$} \\
\hline 96 & (-)-pinoresinol & B. harlandii, B. papuana, B. abbreviata & {$[7,9,26]$} \\
\hline 97 & $(-)$-pinoresinol- $\beta$-D-glucoside & B. japonica, B. abbreviata & {$[25,26]$} \\
\hline 98 & $\begin{array}{l}\text { balaxiflorin } A=\left(7^{\prime} S, 8 R, 8 \text { 'R)-9-O- }\right. \\
\text { galloyllariciresinol-4'-O- } \beta-D-\text { glucopyranoside }\end{array}$ & B. laxiflora & [5] \\
\hline 99 & lariciresinol-4-O- $\beta$-D-glucoside & B. polyandra & {$[15]$} \\
\hline 100 & lariciresinol-4'-O- $\beta$-D-glucoside & B. laxiflora, B. polyandra & {$[5,15]$} \\
\hline 101 & $(-)$-lariciresinol & B. harlandii, B. japonica, B. abbreviata & {$[5,7,15,25,26]$} \\
\hline 102 & balanophonin & B. japonica & {$[25]$} \\
\hline 103 & balanophonin 4-O- $\beta$-D-glucopyranoside & B. japonica & [21] \\
\hline 104 & $7 S, 8 R$ dehydrodiconferyl alcohol & B. latisepala & [30] \\
\hline 105 & 3,3'-bis (3,4-dihydro-6-methoxy-2 H-1-benzopyran) & B. fungosa & {$[16]$} \\
\hline 106 & balajaponin A & B. japonica & [32] \\
\hline 107 & brevifolin & B. simaoensis & {$[28]$} \\
\hline 108 & methyl brevifolincarboxylate & B. involucrata & {$[34]$} \\
\hline 109 & kaempferol & B. spicata & {$[12]$} \\
\hline 110 & quercetin & B. simaoensis & [23] \\
\hline 111 & quercimeritrin & B. simaoensis & [23] \\
\hline 112 & 3-hydroxyphloretin & B. harlandii, B. tobiracola & {$[1,35]$} \\
\hline 113 & hesperetin dihydrochalcone 4'- $\beta$-D-glucoside & B. harlandii, B. tobiracola & {$[1,35]$} \\
\hline 114 & 3-hydroxyphloretin-4'-O- $\beta$-D-glucoside & B. harlandii, B. tobiracola & {$[1,8,35]$} \\
\hline 115 & phloretin 4'-O- $\beta$-D-glucoside & B. harlandii, B. tobiracola & {$[1,8]$} \\
\hline 116 & phloridzin & B. involucrata & [34] \\
\hline 117 & 3-hydroxy-phloridzin & B. involucrata & [34] \\
\hline 118 & (E)-3,4,2',4',6'-pentahydroxychalcone-2'-O- $\beta$-D-glucopyranoside & B. involucrata & [34] \\
\hline 119 & aureusidin-4-O- $\beta$-D-glucopyranoside & B. involucrata & [34] \\
\hline 120 & catechin & B. spicata & [19] \\
\hline 121 & (2R/2 S)-eriodictyol 7-O- $\beta$-D-glucopyranoside & B. harlandii, B. tobiracola, B. involucrata & {$[1,8,36]$} \\
\hline 122 & $\begin{array}{l}\text { (2R/2 S)-eriodictyol (= 2-(3,4-dihydroxyphenyl)- } \\
\text { 2,3-dihydro-5,7-dihydroxy-4 H-1-benzopyran-4-one) }\end{array}$ & B. harlandii, B. involucrata & {$[1,36]$} \\
\hline 123 & (2R/2 S)-eriodictyol 5-O- $\beta$-D-glucopyranoside & B. involucrata & {$[36]$} \\
\hline 124 & eriodictyol 7-(6"-O-galloyl-ß-D-glucoside) & B. harlandii & [1] \\
\hline 125 & monogynol A & B. spicata & [19] \\
\hline 126 & monogynol A 3-palmitate & B. simaoensis & {$[12]$} \\
\hline 127 & lupenoe & B. spicata, B. indica & {$[19,37]$} \\
\hline 128 & lupeol & B. involucrata & {$[16,29,37,38]$} \\
\hline 129 & lupeol acetate & $\begin{array}{l}\text { B. harlandii, B. polyandra, B. involucrata, B. indica, } \\
\text { B. japonica }\end{array}$ & {$[7,16,24,27,29,38,39]$} \\
\hline
\end{tabular}


Table 1 Chemical constituents from genus balanophora (Continued)

\begin{tabular}{|c|c|c|c|}
\hline 130 & Balanophorin B & B. spicata, B. indica, B. simaoensis & {$[19,38,39]$} \\
\hline 131 & $\beta$-amyrin & B. laxiflora, B. involucrata, B. simaoensis & {$[7,29,37,38,40]$} \\
\hline 132 & $\beta$-amyrin acetate & B. spicata, B. indica, B. simaoensis, B. japonica & {$[19,24,39,40]$} \\
\hline 133 & Balanophorin A & B. spicata, B. indica, B. simaoensis, B. involucrata & {$[19,37-39]$} \\
\hline 134 & $\beta$-amyrin stearate & B. simaoensis & [12] \\
\hline 135 & $\beta$-amyrone & B. indica, B. simaoensis & {$[38,39]$} \\
\hline 136 & ursa-12-ene-11-one-3-oloctocosate & B. involucrata & [37] \\
\hline 137 & taraxastenone & B. abbreviata & {$[40]$} \\
\hline \multirow[t]{2}{*}{138} & taraxasterol & B. abbreviata & [31] \\
\hline & & B. indica & \\
\hline 139 & olean-12-ene-3,11-dione & B. tobiracola & [41] \\
\hline 140 & shanzhioside methyl ester & B. spicata & [12] \\
\hline 141 & geniposide & B. spicata & [12] \\
\hline 142 & loganin 6'-O-ß-glucopyranoside & B. latisepala & [30] \\
\hline 143 & clerosterol & B. harlandii & [7] \\
\hline 144 & clerosterol-3-O-(6'-O-palmitoyl)-- $\beta$-D-glucopyranoside & B. harlandii & {$[7]$} \\
\hline 145 & $\beta$-sitosterol & \multicolumn{2}{|c|}{ B. polyandra, B. involucrata, B. simaoensis, B. japonica $[16,24,27,29,37,38]$} \\
\hline 146 & daucosterol & B. simaoensis, B. involucrata & {$[23,29,37]$} \\
\hline 147 & $\beta$-sitosterylglucoside-3'-O-linoleate & B. involucrata & [42] \\
\hline 148 & palmitic acid & B. harlandii, B. simaoensis & {$[7,38,39]$} \\
\hline 149 & 4-hydroxybenzyl- $\beta$-D-glucoside & B. polyandra & [15] \\
\hline
\end{tabular}

that those compounds were a pair of D-glucosyl anomeric isomers $[1,2,14,15]$.

Furthermore, gallic acid (59) and ellagic acid (61) were the part of hydrolysable tannins $[5,7,15,23,24]$.

$2.2 C_{6}-C_{3}$ and $C_{6}-C_{3}-C_{6}$ Constituents. It was reported that about forty-four phenylpropanoids (62-105), three coumarins (106-108) and sixteen flavonoids (109-124) occurred in genus Balanophora, the investigated plants included B. simaoensis, B. spicata, B. involucrata, B. japonica, $B$. harlandii, B. polyandra, B. latisepala, B. laxiflora, B. abbreviata and B. fungosa $[1,5,7,8,15,16,19-21,23-$ 28,30-32,43].

2.2.1 $C_{6}-C_{3}$ Constituents. From 1969, varieties of those constituents were gradually reported from the genus Balanophora [7]. The isolated phenylpropanoids chiefly ranged over simple phenylpropanoids, lignans and coumarins. Numbers of those compounds is from 63 to 106 , with compounds $62-89$ are a term of simple phenylacrylic acid derivatives [1,5,7,14-16,19,23,25-29,32,33,44]. Compound 87 have two coniferin molecules and the location of linkage is explicated to be between $C(9)$ and $C\left(9^{\prime}\right)$. Compounds 89 and 90 possess two coniferin units, and balajaponin $\mathrm{C}(88)$ is the methylated compound 89's aglycon. According to the HMBC and ROESY spectra in balajaponin D (90), the linkage of the two units was assigned to be between $C(6)$ and $C\left(9^{\prime}\right)$, while $C(8)$ and $C\left(9^{\prime}\right)$ in balajaponin $\mathrm{E}$ (89) [32]. The compounds 93-97 were three lignans with a bisepoxy skeleton isolated from B. spicata, B. abbreviata and B. japonica, respectively $[5,7,12,16,25,26]$. (+) -Pinoresinol-di- $O-\beta$ D-glucopyranoside (93) is a glycoside of disaccharide lignan [12,21]. Compounds 98-101 are the monoepoxylignan glucosides with the 9-O-7' framework, while (-) -lariciresinol (101) occupied the opposite configuration compared with others [5,7,15,25,26]. Balaxiflorin A (98) has a galloyl ester group at $\mathrm{C}(9)$ and its $\mathrm{C}(7), \mathrm{C}\left(9^{\prime}\right)$ positions combines an epoxy lignan moiety by an oxygen atom [5]. Balanophonin (102) is a new neo-ligan from the fresh above- and under-ground parts in methanol extract's water-soluble fraction of B. japonica [25]. 3, 3-bis (3, 4dihydro-6-methoxy-2 $H$-1-benzopyran) (105) was from the rhizome of the plant B. fungosa [16].

Compounds 106-108 are coumarins [28,32,34]. Balajaponin A (106) contains a dihydroisocoumarin fragment and one 1, 2, 4-trisubstituted benzene ring based on spectrogram. It was a novel carbon skeleton and some components with this skeleton had been synthesized as pharmaceutical intermediates [32]. The chemical structure 
of methyl brevifolincarboxylate (108) was illuminated based on the analysis of solid-state NMR, and it received considerable interest mainly because of its strong antiviral properties [34].

2.2.2 Flavonoids. Types and quantities of flavonoids and flavonoid glycosides in Balanophora are relatively few (109-124) [1,8,12,19,20,24,35,36,39]. So far, the isolated flavonoids from Balanophora mainly contain flavonol, flavonone, flavanonol, dihydrochalcone and aurone. From 112 to 118 are seven dihydrochalcone compounds and they showed strong inhibitory effects on $\alpha$-glucosidase $[1,8,35,36]$. Aureusidin-4-O- $\beta$-D-glucopyranoside (119) in $B$. involucrata was the only aurone from the genus Balanophora [34].

2.3 Terpenoids. Some pentacyclic triterpenoids (125$139)$ and three iridoids (140-142) were found in the genus Balanophora [12,19,24,31,37-41,45,46]. The investigated plants are mainly B. spicata, B. simaoensis, B. involucrata, $B$. japonica and B. tobiracola. The categories of pentacyclic triterpenoid contain lupinane, oleanane-type and ursane $[7,19,37,39,45]$. Balanophorin A (133) and balanophorin B (130) were two terpenoids ingredients reported from Balanophora by Ultee in 1926 for the first time [38].

2.4 Sterosl. Five sterols (143-147) were isolated from the whole plants of $B$. harlandii and $B$. involucrata $[7,12,16,23,27,29,37,38,42,47]$.

2.5 Others. A palmitic acid (148) was isolated from B. simaoensis, and in B. polyandra 4-hydroxybenzyl- $\beta$-Dglucoside (149) was found $[7,15,38,39]$.

\section{Biological activities of genus Balanophora}

In general, the extracts and the isolated compounds of the genus Balanophora have remarkable free radical scavenging activities, which is the most notable and important pharmacological activities $[1,5,10,15,48]$. Beyond that, they also display inhibiting HIV, hypoglycemic, alcoholic sobering and some other effects [17,20,49-52].

3.1 Radical Scavenging Activities. Many studies had verified that lots of compounds from this genus were effective anti-oxidants, and the hydrolysable tannins exhibited higher activities than that of other compounds $[1,5,15]$. Especially, the compounds with more adjacent phenolic $\mathrm{OH}$ groups (galloyl, pyrogallol, or catechol group) had higher DPPH radical-scavenging activities $[5,15]$.

She et al. reported the acetone extract of the fresh female plant of $B$. laxiflora had obviously radical scavenging activity in DPPH assay $\left(I C_{50}=16.4 \mu \mathrm{g} / \mathrm{ml}\right)$. All the isolated compounds $(8-10,13-15,53-54,59,70,82$, 91-92, 94, 98 and 100) from the plants were evaluated the free-radical-scavenging activities by DPPH assay with ascorbic acid as positive control. In this assay, the hydrolysable tannins presented higher activities than the other kinds of the phenolic compounds, and the balaxiflorin A (98) and balaxiflorin B (82), with galloyl or caffeoyl groups attached, showed stronger activity than matching phenol compounds [5]. $\mathrm{Ho}$ and others convinced the $I C_{50}$ values of $6.0,3.0,6.4$, and $15.7 \mu \mathrm{g} / \mathrm{ml}$ of the crude extract, its derived soluble fractions, EtOAc fraction, $\mathrm{BuOH}$ fraction, and water fraction from male flowers of aforementioned plant in DPPH test, $(+)$-catechin as the reference control. And their $I C_{50}$ values were 5.4, 4.1, $5.8,20.4 \mu \mathrm{g} / \mathrm{ml}$ in superoxide radical scavenging assay (NBT assay), while the $I C_{50}$ of $(+)$-catechin is $9.0 \mu \mathrm{g} / \mathrm{ml}$. Meanwhile, the methanolic extract of the male flower showed stronger DPPH radical scavenging activity and superoxide radical scavenging assay than that of female flower's extract [20].

The free radical scavenging rate of $4 \mathrm{mg} / \mathrm{ml}$ crude extract of B. involucrata was $94.34 \%$. Wang et al. reported that the $80 \%$ acetone extract of $B$. polyandra displayed high free radical scavenging activity with $I C_{50}=14.48 \mu \mathrm{g} /$ $\mathrm{ml}$ [15]. Then, bioassay-guided chemical investigation of the crude extract led to the isolation of 22 phenolic compounds, and they showed striking radical-scavenging activities. The dihydrochalcones 44, 45, 47, 112 and 114, which were found from the $80 \%$ watery acetone extract from the fresh rhizome of $B$. harlandii, with a catechol (= benzene-1,2-diol) moiety as ring $\mathrm{B}$ exhibited higher activities than ascorbic acid [1,52].

DPPH free radical scavenging assay and ferric reducing power assay of the plant B. spicata by Deng et al. revealed that the ethyl acetate extract, $n$-butanol extract, ethanol extract and water-soluble crude polysaccharides had similar anti-oxidatant activities compared with vitamin C. The DPPH experiment (18.91\%) indicated that the scavenging rate was relatively stronger than that of vitamin $C(13.25 \%)$ while the content of ethyl acetate extract was lower than $0.02 \mathrm{mg} / \mathrm{ml}$. When the content of water fraction was $0.06 \mathrm{mg} / \mathrm{ml}$, the scavenging rate of it was $91.08 \%$, which closed to the $95.44 \%$ of vitamin $C$. The $I C_{50}$ of B. spicata ethyl acetate extracts was $6.0 \mu \mathrm{g} / \mathrm{ml}$, while the chloroform extract was the highest [10].

3.2 Inhibit HIV Effects. 1, 2, 6-tri-O-caffeoyl- $\beta$-Dglucopyranose (TCGP) (13) and 1, 3-di-O-caffeoyl-4$O$-galloyl-glucopyranose (DCGGP) (11) were in a dose-dependent relationship in inhibiting the entry of HIV-1 Env pseudovirus into the target cells, with $I C_{50}$ values of $(5.5 \pm 0.2)$ and $(5.3 \pm 0.1) \mu \mathrm{g} / \mathrm{ml}$. The result showed that TCGP and DCGGP were potent HIV-1 entry inhibitors targeting gp41 and could serve as lead ingredients for developing the novel anti-HIV-1 microbicides for prevention of sexual HIV-1 transmission [17]. Sun et al. has convinced that compound 1, 2, 6-tri- $O$-galloyl- $\beta$-Dglucopyranose (TGGP) (22) from B. japonica could inhibit the form of HIV gp41 six-helix bundle formation with an $I C_{50}$ values of $(1.37 \pm 0.19) \mu \mathrm{g} / \mathrm{ml}$. And it inhibited gp41mediated HIV envelope fusion with target cell membrance. The inhibitory activity of TGGP on HIV envelope 
grlycoprotein-induced cell-cell fusion was detected using a non-infectious cell-based assay [48].

3.3 Hypoglycemic Effects. The literature 50 showed that the $95 \%$ ethanol extract of $B$. polyandra could decrease both the fasting and no fasting blood glucose concentrations of ICR mice. The glucose tolerance was improved remarkably in both normal and alloxan-induced diabetic mice. Compared with normal mice, the blood glucose peak of the ethanol extract of $B$. polyandra processed retroposited and declined $40 \%$, the area under the blood glucose-time curve (AUC) declined about 26\%. Trace it to its cause, the inhibition of $\alpha$-glucosidase might be one of its major mechanisms $[6,49]$.

3.4 Anti-inflammatory and Analgesic Effects. Literatures about anti-inflammatory and analgesic effects of plant Balanophora are few. The methanol extracts of B. involucrata had significant anti-inflammatory and analgesic effect. The MTD of it in mice was more than $75 \mathrm{~g} /(\mathrm{kg} \cdot \mathrm{d})$. Hot plate test indicated the healing effect of it was stronger and better than that of diclofenac - a positive control, and the acting time was more durable than morphine's. Acetic acid writhing test showed that both the two doses $(20 \mathrm{~g} / \mathrm{kg}, 12.5 \mathrm{~g} / \mathrm{kg})$ could reduce the number of writhing mice $(P<0.01)$ with the analgesic rates $46.9 \%$ and $39.4 \%$. Meanwhile, it could prolong the time the writhing appeared. According to the result of the experiment, the effect of it was equal to the drug hydrocortisone $[42,47]$.

3.5 Others. Jiang et al. reported that the hydrolyzable tannins 1-O- $(E)$-caffeoyl-6-O- $(S)$-brevifolincarboxyl- $\beta$-Dglucopyranose (31) from $B$. japonica with cytotoxicity to HepG2 cancer cells with $I C_{50}$ values of $4.22 \mu \mathrm{g} / \mathrm{ml}$ [22]. Hosoya et al. found that papuabalanols A (50) showed moderate vasodilator effect on rat aorta, while papuabalanols B (51) had potent inhibition effect of mushroom tyrosinase and anti-melanogenesis in B16 mouse melanoma cells [9]. The (-) -pinoresinol from B. abbreviata had inhibitory activities on the lipopolysaccharide (LPS)-induced inducible nitric oxide synthase (iNOS) expression in RAW 246.7 cells [31]. And extract of B. polyandra could improve the high-fat-diet-induced metabolic syndrome by inhibiting the activity of enzyme PTP1B in mice [13].

\section{Conclusions}

The plants of genus Balanophora have been used as traditional herbal medicines by local people in China. Herein, we systematically expound the multiplex chemical constructions isolated from plants B. simaoensis, B. polyandra, B. spicata, B. fungosa, B. harlandii, B. japonica, B. laxiflora, B. tobiracola, B. papuana, B. abbreviata, B. latisepala, $B$. involucrata and $B$. indica. Studies indicated that the chemical groups of genus Balanophora (Balanophoraceae) were mainly hydrolysable tannins. Cinnamic acid derivative tannins, reported from this genus firstly as a class of novel compounds, are characteristic components in the genus Balanophora. Some polymers of hydrolysable tannin and dihydrochalcone glucoside were reported from B. tobiracola, B. harlandii and B. papuana.

The genus Rhopalocnemis (family Balanophoraceae) possesses the condensed tannins without presence of hydrolysable tannin [53]. Those remarkable chemical characteristic of aforementioned in two genera could provide evidence for their systematic botany [54].

Additionally, oxidative stress has contributed to the development of many human diseases. The use of antioxidants will be a feasible strategy for the treatment of these diseases. And a good many studies have confirmed that the extracts of Balanophora and the isolated compounds possess significant free radical scavenging activities. Some other components still showed the abilities to inhibit HIV and cytotoxicity to cancer cells.

So further studies are necessary to point the chemdiversity and biological significance of these compounds and extend the use of the plants from the genus Balanophora.

\section{Additional file}

Additional file 1: The chemical structure of compounds isolated

from plants of genus Balanophora.

Competing interests

The authors declare that they have no competing interests.

Authors' contributions

$X W, Z L, W Q, G S$ and $B L$ have all been involed in drafting this review. All authors read and approved the final manuscript.

\section{Acknowledgement}

This work was supported by Beijing Nova Program (No. 2011070), and Beijing Key Laboratory of Fundational Research and Drug Development of Traditional Chinese Medicine (TCM).

Received: 12 April 2012 Accepted: 5 July 2012

Published: 1 August 2012

\section{References}

1. Wang W, Zeng SF, Yang CR, Zhang YJ: A new hydrolyzable tannin from Balanophora harlandii with radical-scavenging activity. Helv Chim Acta 2009, 92:1817-1822.

2. Zhang SY: Medicinal plant resources of genus Balanophora. Chinese Journal of Ethnomedicine and Ethnopharmacy 1998, 27-28.

3. Chen JY, Li C, Wang XQ, Zhao ZXZ: Research progress and medicinal plant types of genus Balanophora. Lishizhen Med. Materia Medica Res. 2010, 21:2032-2034.

4. Wu CY: Flora of China. In Editorial Board of 'Flora of China', Chinese Academy of Science. Beijing: Science Press; 1988. 250. 24.

5. She GM, Zhang YJ, Yang CR: Phenolic constituents from Balanophora laxiflora with DPPH radical-scavenging activity. Chem Biodivers 2009, 6:875-880.

6. Wang H, Luo B, Zou K: Chemical constituents and pharmacological studies of genus Balanophora. Lishizhen Med Materia Medica Res 2008, 19:809-811.

7. Teng RW, Wang DZ, Yang CR: Chemical constituents from Balanophora harlandii. Acta Botanica Yunnanica 2000, 22:225-233. 
8. Tanaka T, Uehara R, Nishida K, Kouno I: Galloyl, caffeoyl and hexahydroxydiphenoyl esters of dihydrochalcone glucosides from Balanophora tobiracola. Phytochemistry 2005, 66:675-681.

9. Hosoya T, Nakata A, Zaima K, Latip J, Din LB, Muslim N, Morita H: Papuabalanols $A$ and $B$, new tannins from Balanophora papuana. Chem Pharm Bull 2010, 58:738-741.

10. Deng J, Mo ZC, Ji GQ, Yang J: Antioxidant Activity of Extract from Balanophora spicata Hayata in vitro. Food science 2010, 31:23-25.

11. Wang $H$, Zhang HQ, Wu HY, Zou K: Study of antioxidation effects of extracts of Balanophora involuctata and trans-coniferin. $J$ of China Three Gorges Univ (Natural Science) 2009, 31:99-101.

12. Dai Z: Study of chemical constituents of Balanophora simaoensis and Balanophora spicata. PhD thesis. Beijing University of Chinese Medicine: Chemistry of Traditional Chinese Medicine Department; 2005.

13. Tao RY, Ye F, He YB, Tian JY, Liu GT, Ji TF, Su YL: Improvement of high-fat-diet-induced metabolic syndrome by a compound from Balanophora polyandra Griff mice. Eur J Pharmacol 2009, 616:328-333.

14. Jiang ZH, Hirose Y, Iwata H, Sakamoto S, Tanaka T, Kouno I: Caffeoyl, coumaroyl, galloyl, and hexahydroxydiphenoyl glucoses from Balanophora japonica. Chem Pharm Bull 2001, 49:887-892.

15. Wang KJ, Zhang YJ, Yang CR: New phenolic constituents from Balanophora polyandra with radical-scavenging activity. Chem Biodivers 2006, 3:1317-1324.

16. Panthama N, Kanokmedhakul S, Kanokmedhakul K: Galloyl and hexahydroxydiphenoyl esters of phenylpropanoid glucosides, phenylpropanoids glucosides from rhizome of Balanophora fungosa. Chem Pharm Bull 2009, 57:1352-1355.

17. Xia CL, Mao QC, Li RM, Chen ZM, Jiang SB, Jiang ZH, Liu SW: Study of the mechanism of caffeoyl glucopyranoses in inhibiting HIV-1 entry using pseudotyped virus system. J South Med Univ 2010, 30:720-723.

18. Ogi T, Higa M, Maruyama S: Melanin synthesis inhibitors from Balanophora fungosa. J Agr Food Chem 2011, 59:1109-1114.

19. Dai Z, Wang F, Wang GL, Lin RC: Studies on chemical constituents of Balanophora spicata. Chin J Chin Mat Med 2006, 31:1798-1800.

20. Ho ST, Tung YT, Cheng KC, Wu JH: Screening, determination and quantification of major antioxidants from Balanophora laxiflora flowers. Food Chem 2010, 122:584-588.

21. Jiang ZH, Tanaka T, Iwata H, Sakamoto S, Hirose Y, Kouno I: Ellagitannins and lignan glycosides from Balanophora japonica (Balanophoraceae). Chem Pharm Bull 2005, 53:339-341.

22. Jiang ZH, Wen XY, Tanaka T, Wu SY, Liu ZQ, Iwata H, Hirose Y, Wu SG, Kouno I: Cytotoxic hydrolyzable tannins from Balanophora japonica. J Nat Prod 2008, 71:719-723.

23. Dai Z, Wang GL, Liu Y, Zhang J, Lin RC: The chemical constituents study of Balanophora simaoensis. Chin J Chin Mat Med 2005, 30:1131-1132.

24. Narayanan CR, Kulkarni AK, Quasim C, Wadia MS: Chemical examination of the rhizomes of coffee fungus, Balanophora indica. Indian J Chem 1976, 14B:906-907.

25. Baderschneider B, Winterhalter P: Isolation and characterization of novel benzoates, cinnamates, flavonoids, and lignans from riesling wine and screening for antioxidant activity. J Agr Food Chem 2001, 49:2788-2798.

26. Luo B, Zou K, Wang H, Tang ZC, Yu LL: Studies on chemical constituents of Balanophora involucrata. Li Shizhen Med Materia Medica Res 2007, 18:1929-1930

27. Dai Z, Wang GL, Lin RC: Chemical constituents of Balanophora simaoensis (III). Chin Tradit Herb Drugs 2006, 37:1608-1610.

28. Falshaw CP, Ollis WD, Ormand KL, Mongkolsuk S, Podimuang V: The spectroscopic identification of coniferin. Phytochemistry 1969, 8:913-915.

29. Urones JG, De Pascual Teresa J, Sanchez Marcos I, Dize Martin D: Entisolariciresinol in reseda suffruticosa. Phytochemistry 1987, 26:1540-1541.

30. Haruna M, Koube T, Ito K, Murata H: Balanophonin, a new-lignan from Balanophora japonica Makino. Chem Pharm Bull 1982, 30:1525-1527.

31. Hosokawa A, Sumino M, Nakamura T, Yano S, Sekine T, Ruangrungsi N, Watanabe K, Ikegami F: A new lignan from Balanophora abbreviata and inhibition of lipopolysaccharide (LPS)-induced inducible nitric oxide synthase (INOS) expression. Chem Pharm Bull 2004, 52:1265-1267.

32. Zhou T, Zhang XH, Zhang SW, Liu SS, Xuan LJ: New phenylpropanoids and in vitro a-glucosidase inhibitors from Balanophora japonica. Planta Med 2011, 77:477-481.

33. Pan YJ, Zhou Y, Zou K, Wu J, Li QX, Zhang S: Chemical constituents of Balanophora involucrata. Chin Tradit Herb Drugs 2008, 39:327-331.
34. Pan JY, Zhang S, Yan LS, Tai JD, Xiao Q, Zou K, Zhou Y, Wu J: Separation of flavanone enantiomers and flavanone gluciside diastereomers from Balanophora involucrata Hook. f. By capillary electrophoresis and reversed-phase high-performance liquid chromatography on a $\mathrm{C}_{18}$ column. J Chromatogr A 2008, 1185:117-129.

35. Liu XK, Li ZR, Qiu MH, Nie RL: Triterpene constituents from Balanophora indica. Acta Botanica Yunnanica 1998, 20:369-373.

36. Ito K, Itoigawa M, Haruna M, Murata H, Furukawa H: Dihydrochalcones from Balanophora tobiracola. Phytochemisty 1980, 19:476-477.

37. Chen JY, Li C, Wang XQ, Zhao ZX: The types and research progress of medicinal plants Balanophora. Li Shizhen Med Materia Medica Res 2010, 21:2032-2034.

38. Li F, Li XM, Wang BG: Chemical constituents of marine mangrove plant Bruguiera gymnorrhiza. Marine Scineces 2010, 24:24-27.

39. Xia XZ, Han HX, Tu PF: Studies on triterpenoids and steroids from Balanophora involucrata. Chin Tradit Herb Drugs 2001, 32:6-9.

40. Ruan HL, Zhang YH, Pi HF, Zhao W, Wu JZ: Chemical constituents of Balanophora japonica Makino. Nat Prod Res Dev 2006, 18:74-75.

41. Ruan HL, Li J, Zhao XY, Zhang YH, Pi HF, Wu JZ: Screening the effective fraction of analgesin in Balanophora involucrata. Herald of Medicine 2006, 25:383-384

42. Yagishita K: Isolation and identification of taraxasterol and $\beta$-amyrin from the bird-lime of Balanophora japonica. Bull Agr Chem Soc Japan 1956, 4:206-210.

43. Kanchanapoom T, Picheansoonthon C, Kasai R, Yamasaki K: New glucosides from Thai medicinal plant, Balanophora latisepala. Nat Med 2001, 55:213-216.

44. Xie LH, Akao T, Hamasaki K, Deyama T, Hattori M: Biotransformation of pinoresinol diglucoside to mammalian lignans by human intestinal microflora, and isolation of Enterococcus faecalis strain PDG-1 responsible for the transformation of (+)-pinoresinol to (+)-lariciresinol. Chem Pharm Bull 2003, 51:508-515.

45. Dai Z, Wang GL, Wang F, Ma SC, Lin RC: Chemical constituents from Balanophora simaoensis (I). Chin Tradit Herb Drugs 2005, 36:830-831.

46. Yadagiri B, Raj K, Rao GSRS: Triterpenoids from Balanophora abbreviata and Balanophora indica. J Nat Prod 1984, 47:182.

47. Ruan HL, Li J, Zhao XY, Zhang YH, Xiang M, Wu JZ: Studise on antiinflammatory and analgesic effects of Balanophora involucrata. Chin Arch Tradit Chin Med 2003, 21:910-911.

48. Sun W, Wang HT, Xia CL, Wu SG, Jiang SB, Jiang ZH, Liu SW: 1,2,6-tri-O-galloyl$\beta$-D-glucopyranose inhibits gp41-mediated HIV envelope fusion with target cell membrane. J South Med Univ 2008, 28:1127-1131.

49. Tian JY, Ji TF, Su YL, Cong WN, Liu ZL, Ye F: Studies on hypoglycemic effcet of extract of Balanophora polyandra. Chin J Chin Mat Med 2007, 32:1194-1198.

50. Tang ZC, Zou K, Wang JZ, Ying CZ, Lu HH: Mechanism for the extracts of Tupistra chinensis Bak. and Balanophora involucrata HK. f. to inhibit alcoholism. Li Shizhen Med Materia Medica Res 2007, 18:2958-2960.

51. Zhang Y, Qin LY, Tang SY: Modern research progress of Balanophora involucrata. Journal of China Traditional Chinese Medicine Information 2010, 2:243.

52. Nakamura Y, Watanabe S, Miyake N, Kohno H, Osawa T: Dihydrochalcones: evaluation as novel radical scavenging antioxidants. J Agric Food Chem 2003, 51:3309-3312

53. She GM, Hu JB, Zhang YJ, Yang CR: Phenolic constituents from Rhopalocnemis phalloides with DPPH radical scavenging activity. Pharm Biol 2010, 48:116-119.

54. She GM: Study on polyphenolic constituents and their biological activities from several medicinal herbs and tea-used plants. PhD thesis. Kunming Instituent of Botany: Chinese Academy of Sciences; 2006.

doi:10.1186/1752-153X-6-79

Cite this article as: Wang et al:: Phytochemicals and biological studies of plants from the genus Balanophora. Chemistry Central Journal 2012 6:79. 